\title{
Errata: Analysis of synthetic aperture range image characteristics for seismic disasters in the Wenchuan earthquake
}

\author{
Zhen Li, Quan Chen, Jianmin Zhou, and Bangsen Tian \\ Key Laboratory of Digital Earth Science, Center for Earth Observation and Digital Earth, \\ Chinese Academy of Sciences Beijing 100190, China \\ zli@,ceode.ac.cn
}

This article [J. Appl. Remote Sens. 3, 031685 (2009)] was originally published online on 26 May 2009 with an error in the author list. The first author was listed as "Li Zhang" and should have been "Zhen Li." The author list was corrected on 16 June 2006. 